\title{
Distractors of low activation can produce negative priming
}

\author{
HSUAN-FU CHAO and YEI-YU YEH \\ National Taiwan University, Taipei, Taiwan
}

\begin{abstract}
In two experiments, we examined negative priming produced by distractors of low activation. A hybrid methodology was adopted to incorporate a study phase in which stimuli repetitively served as a target or a distractor, and a test phase in which the relationship in prime-probe couplets was manipulated. A critical manipulation was the presence or absence of novel stimuli in the test phase to alter the list context. The results showed that prime distractors of low activation produced negative priming when the test phase did not include novel items, which provided an optimal retrieval context. Prime distractors of high activation reliably led to negative priming when the list context contained novel items, but the effect varied when the list context did not include novel items. Theoretical implications are discussed.
\end{abstract}

Inhibition pervades cognitive processes (Dagenbach \& Carr, 1994). Inhibitory mechanisms appear to operate only when distracting information imposes strong competition against the target in information processing toward a behavioral goal. In selective attention, this operation principle is demonstrated in the phenomenon of negative priming (Malley \& Strayer, 1995; Strayer \& Grison, 1999). In cued recall tests of memory, this operation principle is demonstrated by the phenomenon of retrieval-induced forgetting (Anderson, Green, \& McCulloch, 2000; Anderson \& Spellman, 1995). In both the selective attention domain and the memory domain, inhibition does not manifest if distracting information is weak in competing against the target of the behavioral goal. Determination of the contingencies required for the operation of inhibition is an important advancement since the work of William James (1890/1950).

James conceptualized selective attention as a mechanism serving two functions: facilitation of target processing and inhibition of distractor processing. Empirical evidence in support of his conceptualization only came much later. Using a Stroop design (Stroop, 1935), Dalrymple-Alford and Budayr (1966) first discovered response suppression in color naming. Color naming was slower when the name of the color was the to-be-ignored word in the preceding trial than when the name was irrelevant. Lowe (1979) replicated this finding and also demonstrated the importance of the presence of a probe distractor. In labeling this phenomenon of delayed target

This research was supported by Grant NSC91-2413-H-002-011 from the National Science Council of Taiwan to Y.-Y.Y. We thank the Ministry of Education Committee for the Promotion and Propagation of the National Language for providing the database from which we selected stimuli. Correspondence concerning this article should be addressed to Y.-Y. Yeh, Department of Psychology, National Taiwan University, No. 1, Sec. 4, Roosevelt Rd., Taipei, 106 Taiwan (e-mail: yyy@ntu.edu.tw). processing by its previous role, negative priming, Tipper (1985) set a milestone for the advancement of research on inhibition in selective attention.

To demonstrate negative priming, prime and probe trials are usually presented in couplets, with the probe trial immediately following its prime trial. The critical manipulation is the relationship between the prime distractor and the probe target. In the ignored repetition (IR) condition, the probe target is identical to the prime distractor. In the control condition, the probe target is neutral with respect to all information in the prime trial. Reaction time in the IR condition is usually slower than the reaction time in the control condition.

The slower response, i.e., negative priming, is assumed to reflect the operation of inhibitory mechanisms as a delay in overcoming the inhibition of the prime distractor (Tipper \& Cranston, 1985). Because negative priming has been demonstrated across a variety of stimuli and tasks (see Tipper, 2001) as a robust effect of inhibition, researchers have used the negative priming paradigm to measure inhibitory deficits for special populations such as the elderly and patients with schizophrenia (for a review, see May, Kane, \& Hasher, 1995).

Negative priming does have its constraints. Malley and Strayer (1995) identified one such constraint by noticing that only a small set of stimuli is used in the majority of negative priming studies. To verify the hypothesis that inhibition only operates when the distractor is a strong competitor, Strayer and Grison (1999) manipulated the strength of competition imposed by the prime distractor. Each stimulus was repeated from zero times to five times either as a target or as a distractor in the trials before becoming a prime distractor. They reasoned that the activation level should be much higher when a stimulus is repeated as a target than when it repetitively serves as a distractor in the trials before becoming the prime distractor. The inhibitory mechanisms should operate in the former con- 
dition but not in the latter, because only a prime distractor of high activation is a strong competitor. Their results indeed supported this prediction. Negative priming manifested when prime distractors were stimuli that had repeatedly appeared as targets in previous trials but not when prime distractors were stimuli that had been repeated as distractors in the previous trials.

Lowe (1998), however, produced results that clearly contradicted Strayer and Grison's (1999) finding. He found significant negative priming in certain experimental contexts for items that repeated as a distractor. Rather than examining negative priming in continuous presentation, Lowe used a memory paradigm in all three experiments with a study phase and a test phase. In the study phase, an item repeated as a target or as a distractor one, three, six, or nine times. All trials were presented randomly in the test phase, rather than in couplets, to examine the priming effect for studied targets and studied distractors. In the test phase of Lowe's second experiment, in which the control condition was excluded, results showed slower response time for studied distractors than for studied targets when both were paired with a novel word. Increasing the number of repetitions further delayed response time for studied distractors.

Negative priming induced by low-activation stimuli greatly challenges the proposition that inhibition only functions on a strong competitor that has high activation. The objective of our study is to resolve the conflicting results of Strayer's studies (Malley \& Strayer, 1995; Strayer \& Grison, 1999) and Lowe's (1998) study. Three major differences in methodology exist between Lowe's study and Strayer and colleagues' studies.

Lowe (1998) used a memory paradigm in which each trial had no relationship with the preceding trial, whereas Strayer and Grison (1999) used a typical negative priming paradigm in which the couplets from the prime and probe trials were used throughout the experiment. Two differences resulted. First, stimulus activation accumulated across a longer interval in Lowe's study than in Strayer and Grison's study. In Lowe's experiments, the test phase followed a study phase of 228 trials and a 5-min retention interval. In Strayer and Grison's Experiment 3B in which the number of repetitions was manipulated, IR and control couplets were preceded by at most 5 trials in which the probe target was presented as the distractor. In their Experiment $3 \mathrm{~A}$, a stimulus served as a target or as a distractor preceding IR and control couplets in zero to two repetitions. There were 3 to 5 trials between each repetition. Thus, the longest interval for a stimulus to accumulate activation was about 10 trials prior to becoming the prime distractor and then the subsequent probe target, a much shorter interval than in Lowe's study.

We consider the difference in the interval for activation accumulation a less likely cause of the contradictory results. Because level of activation should either remain at the same level or decay with retention interval, negative priming should either remain the same or decay to elimination with an increase in retention interval. None of the theoretical accounts would predict the transition from null to significant negative priming by increasing the retention interval. A possible solution is to assume that a short-lived facilitation effect from semantic activation counteracts the inhibition effect in the short-term interval. If we further assume that the inhibition effect lasts longer than the facilitation effect, negative priming is expected to surface after a long retention interval because semantic facilitation is no longer in force. ${ }^{1}$

The second difference in Lowe's use of a memory paradigm as opposed to a typical negative priming paradigm is that the priming score perhaps reflects the operation of different underlying mechanisms. In a typical negative priming paradigm, the priming score is computed in reference to a control condition that has a trial structure identical to that in the IR condition, except that the probe target is unrelated to the prime stimuli. Thus, the priming score mostly reflects the consequence of a probe target's having served as the prime distractor in the preceding trial. Using a memory paradigm, Lowe computed priming scores by comparing the response time for studied distractors with the response time for studied targets when no control condition was included, or with the response time for unstudied new stimuli when a control condition was included. The priming score mostly reflects the influence of the probe target's memory episodes.

Third, as Lowe (1998) noted, negative priming was observed when there was no control condition with novel targets in the test phase. When a control condition was included in his Experiment 1, positive rather than negative priming was observed for both the studied targets and distractors. Lowe suggested that the presence of novel targets in the test phase resulted in context change and thus impaired memory retrieval. In Strayer and Grison's (1999) Experiment 3, in which low-activation distractors did not induce negative priming, novel targets were present throughout the experiment. The experimental context may not have encouraged memory retrieval; without the elicitation of memory retrieval and without the operation of an inhibitory mechanism, a prime distractor with low activation has no effect.

The influence of novel targets is an important hypothesis. Without the presence of novel targets in the test phase, all test targets are highly familiar, and repetition instigates memory retrieval. When novel targets are embedded in the list, their presence offsets the utility of memory retrieval because the items' low memory strength indicates the necessity of stimulus processing. Moreover, any attempt to retrieve novel targets would be slow or terminated due to the failure to recover any appropriate information.

We conducted two experiments using a hybrid method to verify the impact of including novel stimuli on negative priming. Following Lowe's (1998) paradigm, we used a study phase in which each trial was unrelated to the subsequent trial. Each stimulus repeated either as a target or as a distractor in the study phase. Thus, stimu- 
lus activation accumulated over a long interval. Following Strayer and Grison's (1999) paradigm, we used couplets of prime and probe trials in the test phase. Thus, probe targets in the IR condition were recently inhibited. In Experiments 1A and 1B, probe targets were stimuli that repeated six times as studied targets or as studied distractors. In Experiment 1A, novel stimuli were added in the test phase but were absent in Experiment 1B. If low-activation distractors can induce negative priming only when memory retrieval is enhanced, negative priming for low-activation items should be observed only in Experiment 1B, and not in Experiment 1A. We then integrated Experiment 1A and Experiment 1B in Experiment 2 , enabling a within-subjects comparison of negative priming with and without novel items in the test phase.

Because inhibition operates on distractors, the use of couplets is a better context in which to examine the fate of prime distractors with different levels of accumulated activation. A memory method in the test phase can unveil the long-term effect of the consequence of selective attention during the study phase. Yet the absence of a long-term memory of ignored distractors may involve other processes such as memory interference and decay. The use of couplets in the typical negative priming paradigm, in contrast, reveals the immediate aftereffect of selective attention. With a hybrid procedure that allows the accumulation of memory episodes in the study phase and an on-line measure of the aftereffect of selective attention during the test phase, this study should clarify the conditions under which distractors of low activation will produce negative priming.

\section{EXPERIMENT 1}

In addition to the difference in retention interval and in the presence of novel stimuli, the number of repetitions also differed between Lowe's (1998) study and Strayer and Grison's (1999) study. The number of repetitions was one, three, six, or nine times in the former study, and at most five times in the latter study. Lowe found negative priming when the stimulus was repeated as a distractor six or nine times. Thus, it is plausible that stimuli used in Strayer and Grison's study did not repeat enough to elicit inhibition. However, if the absence of novel stimuli is crucial to utilization of memory retrieval, a stimulus that repeats many times as a distractor will not result in negative priming when novel stimuli are present in the list context.

Neill and colleagues (Neill, 1997; Neill \& Mathis, 1998; Neill, Valdes, Terry, \& Gorfein, 1992) have highlighted the importance of memory retrieval in the phenomenon of negative priming. According to the memory retrieval account, negative priming manifests simply because retrieved information from memory traces conflicts with selection of the probe target in the IR condition. For example, when processing a probe target, the retrieved memory trace in which it was a recent distractor is incompatible with the current behavioral goal. Be- cause conflict resolution requires time, it delays response to the current probe target, and negative priming occurs.

Tipper (2001) also emphasized the importance of memory retrieval in negative priming. He suggested two processes crucial for negative priming to manifest. First, a prime distractor's representation must be inhibited by the selection mechanisms. Second, the prime episode must be retrieved by the memory mechanisms in responding to the probe target. When the prime episode is retrieved, the suppressed representation is reinstated, and as a result it slows response selection of the probe target, and negative priming manifests. If the addition of novel targets in the test phase changes the context (Lowe, 1998) such that reinstatement of previous episodes is less likely, the influence of inhibitory mechanisms will not manifest.

The number of repetitions was set to six to incorporate Lowe's (1998) method in the study phase in both Experiments $1 \mathrm{~A}$ and $1 \mathrm{~B}$, with novel stimuli added as fillers in the test phase of Experiment 1A to incorporate Strayer and Grison's (1999) method. The addition of novel targets is assumed to change the list context and to discourage retrieval of previous episodes. No novel stimuli were included in Experiment 1B to set a test phase that encouraged memory retrieval.

If the contradictory findings resulted from the difference in the number of repetitions, with more in Lowe's (1998) study and fewer in Strayer and Grison's (1999) study, Lowe's finding should be replicated in both Experiments $1 \mathrm{~A}$ and $1 \mathrm{~B}$. When we compared the control and IR conditions, negative priming should have manifested, even if a prime distractor was a stimulus that repeated six times as a distractor in the study phase. In contrast, if the contradictory findings resulted from the difference in the test context, negative priming should have been eliminated in Experiment 1A but manifested in Experiment 1B because the test context discourages memory retrieval in the former, but encourages retrieval in the latter. To show that inhibition still functions when the context in the test phase is dissimilar to the study phase, we included a condition in which the prime distractor was studied six times as a target. Repeating as a target accumulates high activation, making the stimulus a strong competitor as a prime distractor (Strayer \& Grison, 1999). This prime distractor should be inhibited such that negative priming manifests in the IR condition.

\section{Method}

\section{Participants}

Thirty-one undergraduate students at National Taiwan University participated in Experiment 1A, and another group of 20 students participated in Experiment 1B for extra credit in an introductory psychology course. All participants were naive to the purpose of the experiment and had normal or corrected-to-normal vision.

\section{Materials and Design}

Using a word count provided by the Ministry of Education Committee for the Promotion and Propagation of the National Language (1997), we selected 150 Chinese character words for Experiment 1A with a count frequency ranging from 252 to 605 per million, and 
with the number of strokes ranging from 5 to 10 . These 150 words were divided into 10 sets and matched in average frequency and number of strokes. Six sets (A-F) were chosen as critical stimuli, and four sets $(\mathrm{G}-\mathrm{J})$ were used as filler sets.

The experiment consisted of two phases: study and test. In the study phase, each trial was unrelated to the previous one, and some stimuli repeated six times as a target, whereas other stimuli repeated six times as a distractor. In the test phase, couplets of prime and probe trials were run continuously. The composition of probe targets in the test phase followed a factorial design of 2 (probe condition: IR or control) $\times 3$ (previous role: target, distractor, novel), using the six sets of critical stimuli. A probe target's previous role was defined by its role in the study phase: target, distractor, or novel (did not occur). In the control condition, the probe target was unrelated to the stimuli in the prime display. In the IR condition, the probe target was the prime distractor from the previous trial. Six forms were generated (see Table 1 for an example) to counterbalance the assignment of each set of critical stimuli. Each set was rotated across participants and served equally often in each cell of the factorial design as the probe targets in the test phase.

In a particular form, study targets were selected from four sets (e.g., A, B, G, J) and study distractors from another four (e.g., C, D, $\mathrm{H}, \mathrm{J})$. Each stimulus word repeated six times, either as a target or as a distractor. When couplets were used in the test phase (see Table 2 for an example), critical stimuli (A-F) were assigned as probe targets in the control or IR condition, while filler stimuli (G-I) were used as prime distractors. Thus, the prime distractors in both the IR and control conditions were equally yoked in the previous role; probe targets in both IR and control conditions were also equivalent in the previous role. Stimuli in filler set J were assigned as both the prime targets and probe distractors in the test phase. Note that the prime target and the probe distractor in each couplet were two different words from set $\mathrm{J}$, but stimuli from this set repeated many times to provide a context of strong competition in the test phase. According to Houghton and Tipper's (1994) computation model, competition is crucial for negative priming to occur.

Because no novel stimuli were used in the test phase of Experiment $1 \mathrm{~B}$, only seven stimulus sets were required. With 16 words in each set, we selected another set of 112 words with a frequency ranging from 252 to 504 per million. From these seven sets, four were selected as critical sets, and the remaining three were designated as filler sets. Four forms were generated to rotate the four critical sets across participants such that each set was assigned equally often in the four conditions (IR/control $\times$ target/distractor) to manipulate probe targets.

A flanker-type display (Eriksen \& Eriksen, 1974) was used in which a target was presented in the center of the screen, flanked by

Table 1

An Example of Assigning Stimulus Materials to Different Conditions in Experiment $1 \mathrm{~A}$

\begin{tabular}{|c|c|c|}
\hline Set & $\begin{array}{l}\text { Role in the } \\
\text { Study Phase }\end{array}$ & Test \\
\hline A & $6 \mathrm{~T}^{*}$ & Probe target in control \\
\hline B & $6 \mathrm{~T}^{*}$ & Probe target in IR \\
\hline $\mathrm{C}$ & $6 \mathrm{D} \dagger$ & Probe target in control \\
\hline $\mathrm{D}$ & $6 \mathrm{D} \dagger$ & Probe target in IR \\
\hline $\mathrm{E}$ & Novel & Probe target in control \\
\hline $\mathrm{F}$ & Novel & Probe target in IR \\
\hline G & $6 \mathrm{~T}^{*}$ & Prime distractor in control of $6 \mathrm{~T}$ \\
\hline $\mathrm{H}$ & $6 \mathrm{D} \dagger$ & Prime distractor in control of 6D \\
\hline I & Novel & Prime distractor in control of novel \\
\hline $\mathrm{J}$ & $6 \mathrm{~T}$ and $6 \mathrm{D} \ddagger$ & Prime target and probe distractor \\
\hline
\end{tabular}

Note-When serving as a probe target in the IR condition, a stimulus is also the prime distractor. *Stimuli studied as targets six times. $\dagger$ Stimuli studied as distractors six times. †Stimuli studied as targets six times and as distractors six times.
Table 2

Example of Couplet Construction in the Test Phase of Experiment 1A

\begin{tabular}{|c|c|c|c|}
\hline \multirow[b]{2}{*}{ Stimulus Type } & \multicolumn{3}{|c|}{ Previous Role in Study Phase } \\
\hline & Target & Distractor & Novel \\
\hline \multicolumn{4}{|c|}{ Ignored Repetition Condition } \\
\hline \multicolumn{4}{|c|}{ gut } \\
\hline Target & $\mathrm{J}$ & $\mathrm{J}$ & $\mathrm{J}$ \\
\hline Distractor & $\mathrm{B}$ & $\mathrm{D}$ & $\mathrm{F}$ \\
\hline \multicolumn{4}{|l|}{ Probe } \\
\hline Target & $\mathrm{B}$ & $\mathrm{D}$ & $\mathrm{F}$ \\
\hline Distractor & $\mathrm{J}$ & $\mathrm{J}$ & $\mathrm{J}$ \\
\hline \multicolumn{4}{|c|}{ Control Condition } \\
\hline \multicolumn{4}{|l|}{ Prime } \\
\hline Target & $\mathrm{J}$ & $\mathrm{J}$ & $\mathrm{J}$ \\
\hline Distractor & G & $\mathrm{H}$ & $\mathrm{I}$ \\
\hline \multicolumn{4}{|l|}{ Probe } \\
\hline Target & A & $\mathrm{C}$ & $\mathrm{E}$ \\
\hline Distractor & $\mathrm{J}$ & $\mathrm{J}$ & $\mathrm{J}$ \\
\hline
\end{tabular}

$\overline{\text { Note-Letters refer to stimulus sets. See Table } 1 \text { for characteristics of }}$ each set.

the same distractor word on both the left and the right sides. The participants viewed the stimuli at a distance of approximately $60 \mathrm{~cm}$. At that viewing distance, each word subtended a visual angle of $0.67^{\circ}$ vertically and $0.67^{\circ}$ horizontally. The center-to-center distance from the target to a distractor was $1.05^{\circ}$ in visual angle. All stimuli were presented in white on a black background.

\section{Procedure}

The experiment was run with DMDX software (Forster \& Forster, 2003). Naming latency was measured with a voice key while the experimenter recorded the accuracy. Each participant was tested individually.

The participants were asked to name the target in the center as quickly and as accurately as possible. They were also told to ignore the two words adjacent to the target because the distractors could interfere with their response. Twelve practice trials were conducted to ensure that the participants understood the instructions. After practice, 360 trials were run in random sequence in the study phase of Experiment $1 \mathrm{~A}$, with a pause after every 60 trials. A test phase immediately followed with 90 prime-probe couplets in random sequence. The participants rested after every 30 prime-probe couplets. In Experiment 1B, 384 trials were run in the study phase with a pause after every 64 trials, and the test phase consisted of 64 couplets. All the experimental trials were run continuously to ensure that the participants were not aware of the distinction between the study phase and the test phase.

The time course of a trial was as follows. A cross in the center of the screen and a "ding" sound were simultaneously presented for $300 \mathrm{msec}$ as a warning signal. A stimulus display was subsequently presented on the screen until the participant spoke the word or until $3 \mathrm{sec}$ had elapsed. The next warning display was presented with a delay of $200 \mathrm{msec}$ after the participant said the word.

The nearby experimenter recorded naming errors. An error was recorded when a participant named the distractor instead of the target. Recording failures, such as when the voice did not register or when the subject coughed, were also recorded but not analyzed.

\section{Results and Discussion}

\section{Primes}

Experiment 1A. One participant's prime and probe data were excluded from further analysis because of a high error rate in the probes (21.7\%). A total of $1.4 \%$ of 
the trials was excluded from data analysis due to recording failures. Performance to a prime was analyzed according to the structure of the subsequent probe trial and the previous role of the prime distractor to confirm that the type of probe trial does not affect the performance to its prime trial. Table 3 shows the average median reaction time (RT), and Table 4 shows the error rate of each condition.

Median RTs were analyzed by a repeated measures analysis of variance (ANOVA) with a 2 (probe condition: IR/control) $\times 3$ (previous role of prime distractor: target/ distractor/novel) within-subjects design. Only the main effect of previous role was significant $[F(2,58)=3.98$, $\left.M S_{\mathrm{e}}=425.60, p<.05\right]$. A post hoc Tukey test showed that naming a prime target was slower when the distractor's previous role was a target than when the distractor's previous role was a distractor $(p<.05)$. Other effects were not significant $(p \mathrm{~s}>.10)$.

Analysis of error rates by the repeated measures ANOVA showed a significant main effect of previous role $\left[F(2,58)=3.20, M S_{\mathrm{e}}=6.05, p<.05\right]$. A post hoc Tukey test showed that target naming was more error prone when flanked by a distractor whose previous role was a target than when the distractor's previous role was a distractor $(p<.05)$. Other effects were not significant $(p \mathrm{~s}>.10)$.

Both RT and error rate data in the prime trials revealed that target naming was more difficult when it was accompanied by high-activation distractors. Thus, items that were repeated as studied targets - namely, high-activation items - interfered with the processing of targets more than low-activation items that were repeated as studied distractors. When prime distractors were novel, responses to prime targets were not significantly different from responses to prime targets accompanied by distractors studied as targets or distractors. The null results suggest that novel distractors impose some degree of interference, perhaps from capturing attention by their novelty (Johnston, Hawley, Plewe, Elliott, \& DeWitt, 1990).

Experiment 1B. A total of $0.5 \%$ of the trials was excluded from data analysis due to recording failures. Table 3

Table 3

Averages and Standard Deviations of Median Correct Reaction Times (in Milliseconds) in Responding to Prime Targets

\begin{tabular}{|c|c|c|c|c|c|c|c|}
\hline \multirow[b]{3}{*}{ Experiment } & \multirow{3}{*}{$\begin{array}{c}\text { Probe } \\
\text { Condition }\end{array}$} & \multicolumn{6}{|c|}{ Previous Role of Prime Distractors } \\
\hline & & \multicolumn{2}{|c|}{ Target } & \multicolumn{2}{|c|}{ Distractor } & \multicolumn{2}{|c|}{ Novel } \\
\hline & & Avg. & $S D$ & Avg. & $S D$ & Avg. & $S D$ \\
\hline \multirow[t]{2}{*}{ 1A: With novel } & IR & 530 & 68 & 518 & 55 & 522 & 54 \\
\hline & Control & 524 & 57 & 516 & 54 & 528 & 52 \\
\hline \multirow[t]{2}{*}{ 1B: No novel } & IR & 531 & 65 & 523 & 59 & & \\
\hline & Control & 533 & 76 & 522 & 65 & & \\
\hline \multirow[t]{2}{*}{ 2: With novel } & IR & 526 & 66 & 513 & 59 & 518 & 57 \\
\hline & Control & 536 & 74 & 516 & 59 & 517 & 70 \\
\hline \multirow[t]{2}{*}{ 2: No novel } & IR & 520 & 77 & 511 & 69 & & \\
\hline & Control & 523 & 74 & 518 & 75 & & \\
\hline
\end{tabular}

Table 4

Averages and Standard Deviations of Error Rates (\%) in Responding to Prime Targets

\begin{tabular}{|c|c|c|c|c|c|c|c|}
\hline \multirow[b]{3}{*}{ Experiment } & \multirow{3}{*}{$\begin{array}{c}\text { Probe } \\
\text { Condition }\end{array}$} & \multicolumn{6}{|c|}{ Previous Role of Prime Distractors } \\
\hline & & \multicolumn{2}{|c|}{ Target } & \multicolumn{2}{|c|}{ Distractor } & \multicolumn{2}{|c|}{ Novel } \\
\hline & & Avg. & $S D$ & Avg. & $S D$ & Avg. & $S D$ \\
\hline \multirow[t]{2}{*}{ 1A: With novel } & IR & 0.9 & 2.8 & 0.2 & 1.2 & 0.5 & 1.7 \\
\hline & Control & 1.8 & 3.5 & 0.2 & 1.2 & 0.7 & 2.0 \\
\hline \multirow[t]{2}{*}{ 1B: No novel } & IR & 1.6 & 3.4 & 0.0 & 0.0 & & \\
\hline & Control & 0.0 & 0.0 & 0.0 & 0.0 & & \\
\hline \multirow[t]{2}{*}{ 2: With novel } & IR & 1.7 & 3.5 & 0.0 & 0.0 & 0.0 & 0.0 \\
\hline & Control & 1.4 & 3.3 & 0.0 & 0.0 & 0.0 & 0.0 \\
\hline \multirow[t]{2}{*}{ 2: No novel } & IR & 2.3 & 3.9 & 0.3 & 1.3 & & \\
\hline & Control & 0.6 & 1.9 & 0.3 & 1.3 & & \\
\hline
\end{tabular}

shows the average median RT, and Table 4 shows the error rate of each condition.

Median RTs were analyzed by a repeated measures ANOVA with a 2 (probe condition: IR/control) $\times 2$ (previous role: target/distractor) within-subjects design. Only the main effect of the previous role was significant $\left[F(1,19)=6.43, M S_{\mathrm{e}}=302.73, p<.05\right]$, showing that naming a prime target accompanied by a distractor that was studied as a target is slower than naming one with a prime distractor that was studied as a distractor. Other effects were not significant $(p s>.10)$.

Analysis of error rates by the repeated measures ANOVA showed that the main effect of couplet type, the main effect of previous role, and their interaction approached significance $\left[F \mathrm{~s}(1,19)=4.13, M S_{\mathrm{e}} \mathrm{s}=2.96, p \mathrm{~s}=.053\right]$. This pattern results from relatively higher error rates when the prime distractor's previous role was a studied target and then served as the probe target in the following IR trial.

The RT data revealed that target naming in the prime trials was more difficult when high-activation distractors accompanied it. Thus, items that have been repeated as studied targets - namely, high-activation items - can interfere with the processing of targets more than lowactivation items that have been repeated as studied distractors. Although only marginally reliable, there was a trend of a higher error rate in the IR condition when the prime distractors were previously studied as targets.

\section{Probes}

Experiment 1A. A total of 3.8\% of the trials was excluded from data analysis due to recording failures or due to errors made in responding to the prime target. Thus, both RT and error rate reflect the performance in correctly naming probe targets contingent upon correct response to the prior prime target. The average median RT and error rate of each condition are presented in Tables 5 and 6 , respectively.

Median RTs were analyzed by a repeated measures ANOVA with a 2 (probe condition: IR/control) $\times 3$ (previous role of probe target: target/distractor/novel) withinsubjects design. The main effect of probe type was not significant $\left[F(1,29)=1.55, M S_{\mathrm{e}}=540.94, p>.10\right]$. The 
Table 5

Averages and Standard Deviations of Median Correct Reaction Times (in Milliseconds) in Responding to Probe Targets

\begin{tabular}{|c|c|c|c|c|c|c|c|}
\hline \multirow[b]{3}{*}{ Experiment } & \multirow{3}{*}{$\begin{array}{c}\text { Probe } \\
\text { Condition }\end{array}$} & \multicolumn{6}{|c|}{ Previous Role of Prime Distractors } \\
\hline & & \multicolumn{2}{|c|}{ Target } & \multicolumn{2}{|c|}{ Distractor } & \multicolumn{2}{|c|}{ Novel } \\
\hline & & Avg. & $S D$ & Avg. & $S D$ & Avg. & $S D$ \\
\hline \multirow[t]{3}{*}{ 1A: With novel } & IR & 523 & 63 & 528 & 53 & 529 & 51 \\
\hline & Control & 510 & 50 & 527 & 50 & 529 & 50 \\
\hline & Difference & $-13^{*}$ & & -1 & & 1 & \\
\hline \multirow[t]{3}{*}{ 1B: No novel } & IR & 533 & 63 & 555 & 69 & & \\
\hline & Control & 522 & 53 & 539 & 57 & & \\
\hline & Difference & -12 & & $-16^{*}$ & & & \\
\hline \multirow[t]{3}{*}{ 2: With novel } & IR & 541 & 63 & 542 & 74 & 548 & 74 \\
\hline & Control & 529 & 69 & 540 & 84 & 544 & 81 \\
\hline & Difference & $-12 *$ & & -2 & & -4 & \\
\hline \multirow[t]{3}{*}{ 2: No novel } & IR & 526 & 72 & 551 & 86 & & \\
\hline & Control & 526 & 70 & 540 & 79 & & \\
\hline & Difference & 0 & & $-12 *$ & & & \\
\hline
\end{tabular}

Note-IR, ignored repetition. $* p<.05$.

main effect of previous role was significant $[F(2,58)=$ $\left.10.15, M S_{\mathrm{e}}=269.37, p<.01\right]$, showing a faster naming time when a probe target's previous role was a target than when its previous role was a distractor or when it had never been studied $(p \mathrm{~s}<.01)$. The two-way interaction approached significance $\left[F(1,29)=2.51, M S_{\mathrm{e}}=314.25\right.$, $p<.10]$. Planned contrasts revealed that negative priming was significant when the previous role of a probe target was a studied target $\left[F(1,87)=6.13, M S_{\mathrm{e}}=389.81, p<\right.$ $.05]$, but not when it was a studied distractor $[F(1,87)=$ $0.04, p>.10]$ or a novel stimulus $[F(1,87)=0.02, p>$ .10]. No effects were significant in the same analysis of error data $(p \mathrm{~s}>.10)$.

Experiment 1B. A total of $2.8 \%$ of the trials was excluded from further data analysis. The average median RT and error rate of each condition are presented in Tables 5 and 6 , respectively.

Median RTs were analyzed by a repeated measures ANOVA with a 2 (probe type) $\times 2$ (previous role) withinsubjects design. Both probe type $\left[F(1,19)=8.83, M S_{\mathrm{e}}=\right.$

Table 6

Averages and Standard Deviations of Error Rates (\%) in Responding to Probe Targets

\begin{tabular}{clrrrrrr}
\hline & & \multicolumn{3}{c}{ Previous Role of Prime Distractors } \\
\cline { 3 - 8 } Experiment & Probe & \multicolumn{2}{c}{ Target } & \multicolumn{3}{c}{ Distractor } & \multicolumn{2}{c}{ Novel } \\
\cline { 3 - 9 } & Condition & Avg. & $S D$ & Avg. & $S D$ & Avg. & $S D$ \\
\hline 1A: With novel & IR & 0.9 & 2.9 & 1.6 & 2.9 & 1.1 & 3.0 \\
& Control & 0.0 & 0.0 & 0.5 & 2.0 & 0.7 & 2.1 \\
& Difference & -0.9 & & -1.1 & & -0.4 & \\
1B: No novel & IR & 0.9 & 2.3 & 4.8 & 5.6 & & \\
& Control & 0.3 & 1.4 & 0.9 & 2.3 & & \\
& Difference & -0.6 & & $-3.7^{*}$ & & & \\
2: With novel & IR & 0.3 & 1.4 & 0.6 & 2.9 & 2.3 & 3.2 \\
& Control & 0.3 & 1.3 & 0.0 & 0.0 & 0.6 & 1.9 \\
& Difference & 0.0 & & -0.6 & & $-1.7^{*}$ & \\
& IR & 1.2 & 2.6 & 1.5 & 3.6 & & \\
& Control & 0.6 & 2.0 & 0.0 & 0.0 & & \\
& Difference & -0.6 & & $-1.5^{*}$ & & & \\
\hline
\end{tabular}

Note-IR, ignored repetition. ${ }^{*} p<.05$.
$421.44, p<.01]$ and previous role $[F(1,19)=28.52$, $\left.M S_{\mathrm{e}}=258.82, p<.01\right]$ were significant in the main effect. The two-way interaction was not significant $[F(1,19)=$ $\left.0.32, M S_{\mathrm{e}}=290.00, p>.20\right]$. Planned contrasts revealed that negative priming was significant only when the probe target's previous role was a studied distractor $\left[F(1,38)=7.00, M S_{\mathrm{e}}=355.72, p<.05\right]$.

Analysis of error rates revealed that both main effects were significant: probe type $\left[F(1,19)=11.80, M S_{\mathrm{e}}=\right.$ $8.47, p<.01]$ and previous role $\left[F(1,19)=9.46, M S_{\mathrm{e}}=\right.$ $10.56, p<.01]$. The two-way interaction was marginally significant $\left[F(1,19)=3.93, M S_{\mathrm{e}}=13.11, p=.058\right]$. Planned contrasts revealed that negative priming was significant when the probe target's previous role was a studied distractor $\left[F(1,38)=13.67, M S_{\mathrm{e}}=10.79, p<\right.$ $.01]$, but not when it was a studied target $[F(1,38)=$ $0.37, p>.10]$.

The results of Experiment 1 show the influence of test context on negative priming. Although the overall naming latency was faster when a probe target had been studied as a target in both Experiments $1 \mathrm{~A}$ and $1 \mathrm{~B}$, negative priming showed different patterns with the presence and absence of novel stimuli. When novel stimuli were present as probe targets in the test phase of Experiment 1A, negative priming was significant only when a probe target's previous role was a studied target. Negative priming was not significant when a probe target's previous role was a studied distractor. The result pattern of Experiment 1B was the opposite.

Assuming that prime distractors are of lower activation when they have been studied as distractors (Strayer \& Grison, 1999), these results are consistent with Lowe's (1998) suggestion that memory retrieval through the manipulation of test context plays an important role in negative priming. Prime distractors of low activation lead to significant negative priming only when the test context encourages memory retrieval by the presence of nothing but the familiar stimuli from the study phase. However, prime distractors of high activation from their repetitive roles as studied targets did not produce significant negative priming when the test context encouraged memory retrieval. They lead to significant negative priming only when the list context includes novel items. This finding causes difficulty for a pure memory retrieval account. According to the memory retrieval account, prime distractors of high activation should produce significant negative priming, especially when the test context encourages memory retrieval. Thus, it is important to replicate the negative priming effect produced by distractors of high activation in the context where novel items are excluded.

\section{EXPERIMENT 2}

We integrated Experiments $1 \mathrm{~A}$ and $1 \mathrm{~B}$ in Experiment 2 to enable a within-subjects comparison of the magnitude of negative priming from low-activation distractors with and without novel items. Following a study phase, two phases were used in the test. Novel stimuli were absent 
in the first phase and were included in the second phase. It was expected that negative priming from distractors of low activation should be observed only when there are no novel targets in the test phase. When novel targets are included in the test phase, negative priming should be found only from prime distractors of high activation.

Because not all participants are likely to detect the change in test context with the presence of novel items, the participants were asked about their awareness of the presence of novel items as a manipulation check. Only data from those who reported awareness were analyzed.

\section{Method}

\section{Participants}

Thirty-one undergraduate students were recruited from an introductory psychology course for extra credit or from an online bulletin board for a monetary reward of NT \$100 (approximately US \$3). All the participants were naive to the purpose of the experiment and had normal or corrected-to-normal vision. Seven of these participants did not indicate an awareness of any new items in the experiment, and their data were excluded from further analysis (their performance is presented in the Appendix).

\section{Design and Materials}

Experiment 2 was a combination of Experiments $1 \mathrm{~A}$ and $1 \mathrm{~B}$, resulting in a 2 (context: with/without novel stimuli) $\times 2$ (probe type: $\mathrm{IR} /$ control) $\times 2$ (previous role: target/distractor) design. The design had four phases: the study phase, the first test phase, the transition phase, and the second test phase. In the study phase, each critical item was studied as either a target or a distractor six times. In the first test phase (the without-novel phase), similar to Experiment $1 \mathrm{~B}$, no novel stimuli were introduced. In the transition phase, novel stimuli were mixed with repeated stimuli to increase the likelihood that participants detected the context change. Finally, in the second test phase (the with-novel phase), similar to Experiment 1A, novel stimuli were presented. The order of the two test phases was constant, with the first phase containing no novel items. The rationale was that it is easier to detect the introduction - rather than the removal — of novel stimuli following a block of repeated stimuli. Furthermore, the continuous flow from the study phase to the first test phase with only the repeated items was expected to encourage memory retrieval.

We selected 18 sets of stimuli with 15 words in each set to fulfill the design of the experiment. The frequency count of these stimuli ranged from 252 to 1,260 per million (Ministry of Education Committee for the Promotion and Propagation of the National Language, 1997), and the number of strokes ranged from 5 to 10 . Eight sets were chosen as critical stimuli, and 10 sets were used as filler sets. These eight critical sets were assigned as probe targets in the eight conditions from the factorial combination: 2 (context) $\times 2$ (probe type) $\times$ 2 (previous role). The assignment was rotated across eight forms.

Two of the filler sets were used as novel probe targets in the second test phase, with one for the IR condition and the other for the control condition. Five filler sets were used as prime distractors in the control conditions to match their previous role to the corresponding IR conditions, with two sets in the first test phase and three in the second test phase when the novel items were added. One filler set was repeated as targets and distractors in the study phase, serving as prime targets and probe distractors in the test phase. Finally, the remaining two filler sets of stimuli were not presented in the study phase and served only as novel prime distractors and novel probe targets in the transition phase.

\section{Procedure}

Because this experiment was a combination of Experiments 1A and $1 \mathrm{~B}$, many aspects are similar. The study phase consisted of 630 trials, with a pause every 70 trials. There were 30 couplets in the transition phase, 60 couplets in the first test phase without novel items, and 90 couplets in the second test phase with novel items. A rest pause occurred every 30 couplets in the latter phases. One important addition to the procedure of Experiments $1 \mathrm{~A}$ and $1 \mathrm{~B}$ is that the participants were asked at the end of the experiment whether they had noticed the introduction of novel stimuli.

\section{Results and Discussion}

\section{Primes}

A total of $0.9 \%$ of the trials was excluded from further data analysis due to recording failures. Table 3 shows the average median RT, and Table 4 shows the error rate of each condition. Performance was analyzed by a 2 (probe type) $\times 5$ (previous role of prime distractor: novel in the with-novel phase/target in the with-novel phase/distractor in the with-novel phase/target in the without-novel phase/ distractor in the without-novel phase) repeated measures ANOVA.

The two-way ANOVA of RT data showed a significant main effect of previous role $\left[F(4,92)=4.77, M S_{\mathrm{e}}=\right.$ $487.70, p<.01]$. A Tukey's test showed a slower RT to a prime target flanked by distractors whose previous roles were targets in the test phase with novel items, than the other three conditions when the prime distractors' previous roles were distractors in either test phase, or when prime distractors were novel items $(p \mathrm{~s}<.05)$. Other effects were not significant $(p \mathrm{~s}>.10)$.

The two-way ANOVA of error rate showed a significant main effect of previous role $\left[F(4,92)=5.59, M S_{\mathrm{e}}=\right.$ $5.19, p<.01]$. A Tukey's test showed a higher error rate to a prime target flanked by distractors whose previous roles were targets in either test phase, than when flanked by distractors whose previous roles were distractors or novel items in the test phase with novel items $(p \mathrm{~s}<.05)$. Other effects were not significant ( $p$ s $>.10)$.

The RT and error rate analyses demonstrated that it is more difficult to name a target when it is accompanied by a distractor studied as a target, than by a distractor studied as a distractor or a novel distractor. Thus, high-activation distractors interfere more with target processing.

\section{Probes}

A total of $2.4 \%$ of the trials were excluded from further data analysis due to recording failures or due to errors made in responding to the prime display. Tables 5 and 6 present the results.

Although novel stimuli occurred only in the second test phase to change list context, a 2 (probe type) $\times 5$ (previous role: novel in the with-novel phase/target in the with-novel phase/distractor in the with-novel phase/target in the without-novel phase/distractor in the withoutnovel phase) repeated measures ANOVA was first conducted to examine whether negative priming was significant from novel stimuli. RT results showed a significant main effect of probe type $\left[F(1,23)=6.12, M S_{\mathrm{e}}=348.45\right.$, $p<.05]$, revealing negative priming. The main effect of previous role was also significant $\left[F(4,92)=4.56, M S_{\mathrm{e}}=\right.$ 
717.57, $p<.01]$. The planned comparison revealed that novel stimuli did not lead to negative priming $(p>.10)$.

The same analysis on error rate data showed a significant main effect of probe type $\left[F(1,23)=6.71, M S_{\mathrm{e}}=\right.$ $6.82, p<.05]$, revealing negative priming. The main effect of previous role approached significance $[F(4,92)=$ $\left.2.21, M S_{\mathrm{e}}=4.87, p=.074\right]$. Moreover, the planned comparison showed that novel stimuli led to negative priming $\left[F(1,115)=8.02, M S_{\mathrm{e}}=4.37, p<.01\right]$. That is, negative priming was not significant from novel stimuli in RT but was significant in the error data.

To clarify the influence of test context, a 2 (context) $\times$ 2 (probe type) $\times 2$ (previous role) repeated measures ANOVA was conducted for both RT and error data. The results from RT data showed a significant main effect of probe type $\left[F(1,23)=5.36, M S_{\mathrm{e}}=628.33, p<.05\right]$, revealing negative priming. The main effect of previous role was also significant $\left[F(1,23)=8.03, M S_{\mathrm{e}}=377.44\right.$, $p<.01]$, confirming that target naming was faster when a probe target's previous role was a target. The context $X$ previous role interaction was significant $[F(1,23)=$ $\left.6.14, M S_{\mathrm{e}}=318.62, p<.05\right]$. Furthermore, the threeway interaction was significant $\left[F(1,23)=4.61, M S_{\mathrm{e}}=\right.$ $301.05, p<.05]$. Planned contrasts revealed that when there were no novel items in the test phase, negative priming was significant only when the prime distractor's previous role was a distractor $\left[F(1,92)=4.20, M S_{\mathrm{e}}=\right.$ $374.09, p<.05]$; when there were novel items in the test phase, negative priming was significant only when a prime distractor had been studied as a target $[F(1,92)=$ $4.85, p<.05]$.

The analysis of error data showed similar results. The main effect of the probe type was significant $[F(1,23)=$ $\left.5.60, M S_{\mathrm{e}}=3.79, p<.05\right]$, revealing negative priming. No other effects were significant $(p \mathrm{~s}>.10)$. Planned contrasts revealed that when there were no novel items in the test phase, negative priming was significant only when a prime distractor had been studied as a distractor $\left[F(1,92)=6.91, M S_{\mathrm{e}}=3.80, p<.01\right]$, but not significant in the other conditions $(p s>.10)$.

The results of Experiment 2 replicated the critical findings with a within-subjects design: Prime distractors of low activation led to negative priming only when there were no novel items in the test phase. Thus, the manifestation of negative priming is not limited to the use of highly activated items, as Strayer and colleagues suggested (Malley \& Strayer, 1995; Strayer \& Grison, 1999).

\section{GENERAL DISCUSSION}

Our results replicated the finding that negative priming manifests mostly when prime distractors are of high activation in the test context with novel items (Malley \& Strayer, 1995; Strayer \& Grison, 1999). The results also replicated Lowe's (1998) finding that prime distractors of low activation can lead to negative priming when the test phase consists of nothing but studied stimuli. The change of list context in the test phase indeed alters the result patterns.

The results showed more than replication of previous findings. Reaction time data showed the absence of negative priming with novel distractors, as Strayer and colleagues found (Malley \& Strayer, 1995; Strayer \& Grison, 1999). Yet novel prime distractors led to a significant negative priming in error rate in Experiment 2, and a trend in Experiment $1 \mathrm{~A}$ showed more errors in the IR condition than in the control condition. Another important observation is the negative priming effect produced by distractors of high activation in different list contexts. In the context where novel items were included in the test phase to discourage memory retrieval, negative priming was significant when prime distractors had been studied as targets. In contrast, the high-activation distractors did not lead to significant negative priming when the test phase did not include novel items to encourage memory retrieval.

The finding that test context can influence negative priming is not without precedence. Kane, May, Hasher, Rahhal, and Stoltzfus (1997) used perceptually degraded probe targets to encourage memory retrieval and observed a larger negative priming effect. Thus, the magnitude of negative priming can be enhanced when the test context encourages processing of the probe target by memory retrieval. With this retrieval mode, it is more likely that the probe target activates its recent episode in which the target was a distractor, albeit one of low activation.

A pure inhibition account has difficulty explaining the results from the change of test context in the observation of significant negative priming produced by distractors of low activation. According to the strong version of the inhibition account (Malley \& Strayer, 1995; Strayer \& Grison, 1999), only distractors of high activation can produce negative priming, with the magnitude increasing with the activation level. Negative priming results because the inhibition mechanisms suppress the activation of distractor representation during the prime trial such that the suppressed representation takes longer to win the race during the probe trial (Houghton \& Tipper, 1994). Distractors of low activation, by this account, should not produce negative priming.

Neill and Joordens (2002), in a pure memory account, proposed that two forces counteract to eliminate negative priming from nonrepeated stimuli that have low activation (but see Strayer, Drews, \& Albert, 2002, for a different view). According to Neill and Joordens's conceptualization, distractors of low activation receive a larger increment in activation than distractors of high activation during the prime trial. The larger increment facilitates processing when these low-activation distractors become probe targets, and hence counteracts the retrieval of inappropriate information from the most recent episode in the prime trial.

Thus, a pure memory model can account for the influence of test context by proposing that the facilitation 
effect and the retrieval effect are in equilibrium to eliminate negative priming for prime distractors of low activation when the test context includes novel items. When the test phase does not include any novel items, memory retrieval dominates the processing. With this dominance, retrieval of inappropriate response information from the most recent prime trial wins the contest over facilitation. Thus, distractors of low activation lead to significant negative priming.

Following the same logic, a pure memory model, however, has difficulty explaining the finding of negative priming in error rate when prime distractors are novel stimuli. A novel prime distractor should receive a larger activation increment than distractors of low activation. If a facilitation effect by activation can offset the retrieval effect for distractors of low activation in the test context with novel items, it should also cancel out the retrieval effect for novel distractors that become probe targets. Yet novel distractors led to higher error rates in the probe trials.

A pure memory retrieval account also has difficulty explaining why negative priming produced by high-activation distractors was unstable when memory retrieval was encouraged. According to this account, prime distractors of high activation receive little incremental activation during the prime trial and hence little priming when becoming probe targets (Neill \& Joordens, 2002). Without activation to counteract the retrieval of inappropriate information from the recent prime episode, high-activation distractors should easily produce negative priming, especially when the test context encourages memory retrieval. Yet negative priming was either marginally significant or not significant when the test context did not include novel items to encourage memory retrieval.

A dual-mechanism hypothesis can account for most of the results observed in this study. Kane et al. (1997) and May et al. (1995) proposed a dual-mechanism account and stated that there are two sources of negative priming: inhibition and memory retrieval, either of which can lead to negative priming. When the list context contains novel items, memory retrieval is not instigated, because it provides little utility for novel items. The memory mechanisms may not be in full operation in this test context. Thus, the results reflect the working of inhibitory mechanisms. When the list context contains nothing but familiar items from the study phase, memory retrieval is instigated. Thus, the change in list context alters the manifestation of the negative priming effect.

For prime distractors of low activation, it is conceivable that inhibitory mechanisms do not suppress the representations of the distractors in the selection for prime trials. Yet a tag of no response is stored in the episodic trace. The inappropriate response information will not affect performance without the reinstatement of this trace. Novel distractors appear to capture attention (Johnston et al., 1990) to interfere with the processing of the prime target as found in the prime data. The capture of atten- tion may have instigated inhibitory mechanisms to produce higher errors and hence significant negative priming. Further investigation is required to understand why this effect surfaces in errors but not in RT data. For lowactivation distractors, no such capture occurs, and memory retrieval is discouraged when novel items are included in the list. Because the representations of these distractors are not suppressed, their activation is not weaker than that of the probe distractors in the selection race of the probe trial. Thus, distractors of low activation do not produce significant negative priming when the list context contains novel items. When the list context contains nothing but familiar items, memory retrieval is instigated, and the inappropriate response information is brought about to interfere with the processing such that negative priming manifests.

For distractors of high activation, inhibitory mechanisms suppress their representations to protect the processing of the prime target. Their activation is lower than that of probe distractors in the selection process. When the test context discourages memory retrieval and reflects the working of inhibitory mechanisms, response to these suppressed representations is slower to produce significant negative priming. When the test context encourages memory retrieval, traces from both the study phase and the prime trial appear to be retrieved. The composite episodic traces increase activation to offset the suppression. Thus, negative priming becomes not significant in the list context that encourages memory retrieval. The results of a preliminary experiment, showing that prime distractors studied nine times as targets produced significant negative priming regardless of list context, suggest that whether the composite episodic traces can counteract suppression may depend on the degree of inhibition.

In his integrated model, Tipper (2001) also pointed out that both the inhibition component and the retrieval component are critical to negative priming. According to Tipper, selective mechanisms are responsible for distractor inhibition during the encoding stage of the prime trial, and retrieval mechanisms utilize prior episodes during the retrieval process of the probe target. Thus, both inhibition and retrieval mechanisms influence the manifestation of negative priming.

The dual-mechanism model and the integrated model differ subtly. The dual-mechanism model considers inhibition and memory retrieval to be two separate sources that do not necessarily relate to each other. The integrated model, on the other hand, considers inhibition and memory retrieval to be two stages of one stream of information processing. In the integrated model, the retrieval of inhibited representations causes negative priming. Thus, the integrated model should predict that whatever happens during the encoding stage should cascade its impact downstream to the retrieval stage.

Because the integrated model considers memory retrieval of suppressed representations to be crucial in the 
manifestation of negative priming, this model also faces difficulty in accounting for the change in the negative priming effect produced by the high-activation distractors as a function of list context. If memory retrieval can bring out the suppressed representations for distractors of low activation, it should also bring out the suppressed representations for distractors of high activation.

With both a pure memory model and the dual-mechanism hypothesis proposing two forces in the observation of negative priming, future endeavors in the behavioral study of negative priming face a greater challenge. The two camps differ by postulating the working of inhibition mechanisms due to response selection in the latter models and by suggesting the retrieval of inappropriate response information in the former models. It may be more difficult to design a critical experiment to falsify one over the other with behavioral data. Another issue that remains to be resolved for the dual-mechanism accounts is whether retrieval and inhibition mechanisms are dissociable. The integrated model predicts no dissociation between inhibition-based negative priming and retrieval-based negative priming. That is, if there is no inhibition during the encoding stage of the prime trials, there should be no negative priming in the retrieval stage of the probe trials.

The results of this study suggest that multiple mechanisms operate in the manifestation of negative priming. Determining the nature of these mechanisms remains a challenge for future research with five factors to be considered: (1) activation accumulated as a study item, (2) activation received as a distractor in the most recent prime episode, (3) retrieval of inappropriate response information or suppressed representation from the recent episode, (4) retrieval of studied episodes, and (5) test context. All of these factors can alter the manifestation of negative priming.

\section{REFERENCES}

Anderson, M. C., Green, C., \& McCulloch, K. C. (2000). Similarity and inhibition in long-term memory: Evidence for a two-factor theory. Journal of Experimental Psychology: Learning, Memory, \& Cognition, 26, 1141-1159.

Anderson, M. C., \& Spellman, B. A. (1995). On the status of inhibitory mechanisms in cognition: Memory retrieval as a model case. Psychological Review, 102, 68-100.

DAGENBACH, D., \& CARR, T. H. (EDS.) (1994). Inhibitory processes in attention, memory, and language. San Diego: Academic Press.

Dalrymple-Alford, E. C., \& Budayr, B. (1966). Examination of some aspects of the Stroop color-word test. Perceptual \& Motor Skills, 23, 1211-1214.

ERIKSEN, B. A., \& ERIKSEN, C. W. (1974). Effects of noise letters upon the identification of a target letter in a nonsearch task. Perception \& Psychophysics, 16, 143-149.

Forster, K. I., \& Forster, J. C. (2003). DMDX: A Windows display program with millisecond accuracy. Behavior Research Methods, Instruments, \& Computers, 35, 116-124.

Houghton, G., \& Tipper, S. P. (1994). A model of inhibitory mechanisms in selective attention. In D. Dagenbach \& T. H. Carr (Eds.), Inhibitory processes in attention, memory, and language (pp. 53-112). San Diego: Academic Press.

JAMES, W. (1950). The principles of psychology. New York: Dover. (Original work published 1890)

Johnston, W. A., Hawley, K. J., Plewe, S. H., Elliott, J. M. G., \& DEWITT, M. J. (1990). Attention capture by novel stimuli. Journal of Experimental Psychology: General, 119, 397-411.

Kane, M. J., May, C. P., Hasher, L., RahHal, T., \& Stoltzfus, E. R. (1997). Dual mechanisms of negative priming. Journal of Experimental Psychology: Human Perception \& Performance, 23, 632-650.

LowE, D. G. (1979). Strategies, context, and the mechanism of response inhibition. Memory \& Cognition, 7, 382-389.

LowE, D. [G.] (1998). Long-term positive and negative identity priming: Evidence for episodic retrieval. Memory \& Cognition, 26, 435443.

Malley, G. B., \& Strayer, D. L. (1995). Effect of stimulus repetition on positive and negative identity priming. Perception \& Psychophysics, 57, 657-667.

MaY, C. P., KANE, M. J., \& HaSher, L. (1995). Determinants of negative priming. Psychological Bulletin, 118, 35-54.

Ministry of Education Committee for the Promotion and PropAGATION OF THE NATIONAL LANGUAGE (1997). The frequency statistics of the words and phrases in the compiled materials of the Concise Mandarin Dictionary. Taipei, Taiwan: Author.

NeILL, W. T. (1997). Episodic retrieval in negative priming and repetition priming. Journal of Experimental Psychology: Learning, Memory, \& Cognition, 23, 1291-1305.

NeILL, W. T., \& JoORDENS, S. (2002). Negative priming and multiple repetition: A reply to Grison and Strayer (2001). Perception \& Psychophysics, 64, 855-860.

NeILl, W. T., \& MATHIs, K. M. (1998). Transfer-inappropriate processing: Negative priming and related phenomena. In D. L. Medin (Ed.), The psychology of learning and motivation (Vol. 38, pp. 1-44). San Diego: Academic Press.

Neill, W. T., Valdes, L. A., Terry, K. M., \& Gorfein, D. S. (1992). Persistence of negative priming: II. Evidence for episodic trace retrieval. Journal of Experimental Psychology: Learning, Memory, \& Cognition, 18,993-1000.

Strayer, D. L., Drews, F. A., \& Albert, R. W. (2002). Negative priming and stimulus repetition: A reply to Neill and Joordens (2002). Perception \& Psychophysics, 64, 861-865.

STRAYER, D. L., \& GRISON, S. (1999). Negative identity priming is contingent on stimulus repetition. Journal of Experimental Psychology: Human Perception \& Performance, 25, 24-38.

STROOP, J. R. (1935). Studies of interference in serial verbal reactions. Journal of Experimental Psychology, 18, 643-662.

TIPPER, S. P. (1985). The negative priming effect: Inhibitory priming by ignored objects. Quarterly Journal of Experimental Psychology, 37A, 571-590.

TIPPER, S. P. (2001). Does negative priming reflect inhibitory mechanisms? A review and integration of conflicting views. Quarterly Journal of Experimental Psychology, 54A, 321-343.

TIPPER, S. P., \& CRANSTON, M. (1985). Selective attention and priming: Inhibitory and facilitatory effects of ignored primes. Quarterly Journal of Experimental Psychology, 37A, 591-611.

\section{NOTE}

1. We thank Daniel Holender for suggesting this possibility. 


\section{APPENDIX}

Averages and Standard Deviations of Median Correct Reaction Times (RTs, in Milliseconds) and Error Rates (ER, in \%) of Probe Trials from 7 Participants Who Did Not Notice Novel Stimuli in Experiment 2

\begin{tabular}{|c|c|c|c|c|c|c|c|c|c|c|c|c|}
\hline \multirow{4}{*}{$\begin{array}{c}\text { Probe } \\
\text { Condition }\end{array}$} & \multicolumn{12}{|c|}{ Previous Role of Probe Targets in the Study Phase } \\
\hline & \multicolumn{4}{|c|}{ Target } & \multicolumn{4}{|c|}{ Distractor } & \multicolumn{4}{|c|}{ Novel } \\
\hline & \multicolumn{2}{|c|}{ RT } & \multicolumn{2}{|c|}{ ER } & \multicolumn{2}{|c|}{ RT } & \multicolumn{2}{|c|}{ ER } & \multicolumn{2}{|c|}{ RT } & \multicolumn{2}{|c|}{ ER } \\
\hline & Avg. & $S D$ & Avg. & $S D$ & Avg. & $S D$ & Avg. & $S D$ & Avg. & $S D$ & Avg. & $S D$ \\
\hline \multicolumn{13}{|c|}{ With Novel } \\
\hline IR & 503 & 50 & 1.0 & 2.3 & 533 & 66 & 1.9 & 3.0 & 529 & 51 & 1.9 & 3.0 \\
\hline Control & 530 & 45 & 0.0 & 0.0 & 514 & 59 & 0.0 & 0.0 & 530 & 47 & 1.0 & 2.5 \\
\hline Difference & $28 *$ & & -1.0 & & -19 & & -1.9 & & 2 & & -0.9 & \\
\hline \multicolumn{13}{|c|}{ Without Novel } \\
\hline IR & 489 & 44 & 1.0 & 2.3 & 505 & 64 & 3.0 & 5.2 & & & & \\
\hline Control & 483 & 38 & 1.0 & 2.5 & 495 & 51 & 2.1 & 3.3 & & & & \\
\hline Difference & -6 & & 0.0 & & -10 & & -0.9 & & & & & \\
\hline
\end{tabular}

Note-IR, ignored repetition. $* p<.05$.

(Manuscript received January 4, 2002;

revision accepted for publication January 22, 2004.) 